02

\title{
Зонно-энергетическая структура и оптические спектры кристаллов $\mathrm{LiNaSO}_{4}$
}

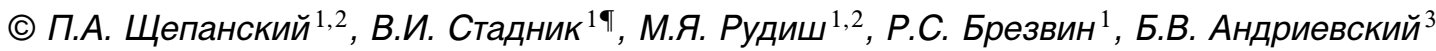 \\ 1 Львовский национальный университет им. Ивана Франко, \\ 79005 Львов, Украина \\ ${ }^{2}$ Академия имени Яна Длугоша в Ченстохове, \\ 42200 Ченстохова, Польша \\ ${ }^{3}$ Кошалинский технологический университет, \\ 75453 Кошалин, Польша \\ ฯ e-mail: vasylstadnyk@ukr.net
}

Поступила в редакцию 23.04.2018 г.

В рамках теории функционала плотности рассчитана зонно-энергетическая структура кристалла $\mathrm{LiNaSO}_{4}$ для пространственной группы P31c. Установлено, что ширина запрещенной зоны $E_{g}$ в приближении GGA составляет $5.49 \mathrm{eV}$. Вершина валентной зоны сформирована в основном р-электронами кислорода, а дно зоны проводимости - $s$-состояниями лития и натрия. Рассмотрено влияние катионного замещения $\mathrm{Na} \rightarrow \mathrm{K} \rightarrow \mathrm{Rb} \rightarrow \mathrm{NH}_{4}$ на электронную структуру кристаллов группы $\mathrm{LiBSO}_{4}$. На основе рассчитанной диэлектрической функции получены спектральные зависимости показателей преломления и коэффициента поглощения кристалла и проведено их сравнение с экспериментальными данными.

DOI: $10.21883 /$ OS.2018.09.46547.115-18

\section{Введение}

Кристалл литий натрий сульфата (ЛНС) $\mathrm{LiNaSO}_{4}$ принадлежит к группе сульфатов $\mathrm{LiBSO}_{4}$, где $\mathrm{B}=$ $=\mathrm{Li}, \mathrm{Na}, \mathrm{K}, \mathrm{Rb}, \mathrm{Cs}, \ldots$, которые долгое время привлекают внимание исследователей из-за способности испытывать ряд фазовых превращений и при этом отличаются ферроэлектрическими, сегнетоэластическими, а также суперионными свойствами. Для $\mathrm{LiNaSO}_{4}$ характерны две фазы: низкотемпературная $\beta$-фаза и высокотемпературная $\alpha$-фаза с фазовым переходом при $\sim 788 \mathrm{~K}[1]$. Кристаллы $\alpha$-ЛНС имеют кубическую объемо-центрическую структуру и обладают быстрой ионной проводимостью. Для $\beta$-ЛНС характерна тригональная симметрия, соответствующая пространственной группе $P 31 c$, и наблюдаются пироэлектрический свойства. При комнатной температуре параметры решетки ЛНС: $a=7.6270 \AA, c=9.8579 \AA \AA$, плотность составляет $2.527 \mathrm{~g} / \mathrm{cm}^{3}$ [2].

Физические свойства кристалла $\mathrm{LiNaSO}_{4}$ довольно хорошо изучены. Много внимания уделено исследованию структуры [1-3], электрических [4], термических [1,5], люминесцентных [6] свойств, ядерного магнитного резонанса [7], а также инфракрасных спектров и спектров комбинационного рассеяния света [8]. Такой интерес к кристаллу связан в частности с суперионными свойствами в $\alpha$-фазе и особенностями фазового перехода $\beta \rightarrow \alpha$. Недавно исследован также край фундаментального поглощения кристалла $\beta$ - $\mathrm{LiNaSO}_{4}$ [9], где установлено, что наиболее вероятным типом оптических переходов вблизи края поглощения кристалла является непрямой разрешенный переход. Ширина запрещенной зоны $E_{g}$ ЛНС равна $4.7 \mathrm{eV}$. Несмотря на значительный инте- рес к физическим свойствам ЛНС на данный момент какие-либо данные об зонно-энергетической структуре кристалла отсутствуют.

Ранее исследованы рефрактивные свойства кристалла $\mathrm{LiNaSO}_{4}[10]$. Интерес к ним связан с тем, что кристаллы группы $\mathrm{ABSO}_{4}$ часто обладают явлением инверсии знака двулучепреломления [11-13], которое находит практическое применение в узкозонных оптических фильтрах, при определении точного состава вещества, в датчиках температуры и давления [14-16].

Цель настоящей работы - исследование электронной структуры и оптических спектров кристаллов $\mathrm{LiNaSO}_{4}$ с использованием первопринципных расчетов $a b$ initio, a также сравнение с экспериментальными результатами.

\section{Методика расчетов}

Расчеты зонно-энергетической структуры проводились самосогласованно с использованием формализма КонаШэма в рамках теории функционала плотности (DFT) с помощью программы CASTEP [17]. Исходными параметрами были данные рентгеноструктурного анализа. В качестве базиса использовались плоские волны. Для описания обменно-корреляционного взаимодействия задействован обменно-корреляционный функционал в виде обобщенного градиентного приближения (GGA) с параметризацией Пердью-Бурке-Эрнцерхофа (PBE) и приближения локальной плотности (LDA). Электроны остова рассматривались в виде ультрамягкого псевдопотенциала Вандербильта. Электронная конфигурация: $\mathrm{O}\left[2 s^{2} 2 p^{4}\right] \quad \mathrm{S}\left[3 s^{2} 3 p^{4}\right] \quad \mathrm{Li}\left[1 s^{2} 2 s^{1}\right] \quad \mathrm{Na}\left[2 s^{2} 2 p^{6} 3 s^{1}\right]$. Предельная кинетическая энергия плоских волн ограни- 
чивалась в расчетах значением $370 \mathrm{eV}$. Интегрирование по $k$-точкам зоны Бриллюэна (ЗБ) проводилось по сетке Монхроста-Пака $2 \times 2 \times 2$.

Перед расчетами проводилась оптимизация элементарной ячейки и положения атомов с помощью алгоритма BFGS (Бройдена-Флетчера-Голдфарба-Шанно). Параметры, использованные для вычислений, выбирались следующим образом. Критерием остановки самосогласованного цикла было достижение сходимости энергии системы с погрешностью $E=10^{-6} \mathrm{eV} /$ Atom. Критерием получения оптимальной геометрии структуры были следующие параметры: сходимость энергии $10^{-5} \mathrm{eV} /$ Atom, максимальная сила $0.03 \mathrm{eV} / \AA$, максимальное давление $0.05 \mathrm{GPa}$, максимальное смещение $10^{-3} \AA$.

Структура ЗБ, используемая для построения диаграммы энергетических зон, представлена на рис. 1.

\section{Результаты и их обсуждение}

\section{Зонно-энергетическая структура кристаллов $\mathrm{LiNaSO}_{4}$}

Элементарная ячейка кристалла ЛНС представлена на рис. 2. Структура кристалла базируется на тетраэдрах $\mathrm{SO}_{4}^{2-}$, что свойственно для сульфатов щелочных металлов. Данные анионные комплексы образуют тетраэдрические и октаэдрические пустоты, в которых размещены ионы $\mathrm{Li}^{+}$и $\mathrm{K}^{+}$соответственно.

Для расчетов использованы данные проведенного нами структурного анализа кристаллов, для которых проводились исследования рефрактивных свойств [10]. Экспериментально полученные параметры элементарной ячейки и оптимизированные методом BFGS представлены в таблице. Как видно из таблицы, значения, оптимизированные с использованием функционала LDA, являются несколько меньше экспериментальных (макси-

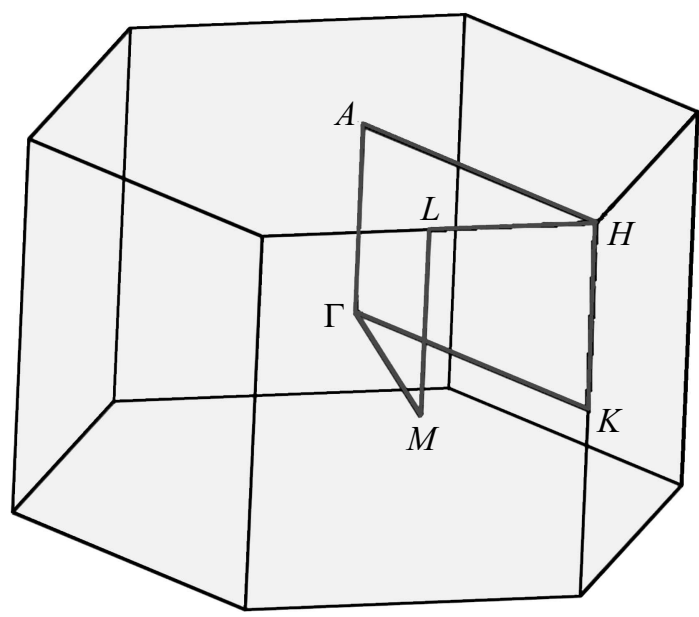

Рис. 1. Зона Бриллюэна для кристаллов $\mathrm{LiNaSO}_{4}$. Координаты специальных точек: $\Gamma(0,0,0), \mathrm{A}(0,0,0.5), \mathrm{H}(-0.33,0.667,0.5)$, $\mathrm{K}(-0.33,0.667,0), \mathrm{M}(0,0.5,0), \mathrm{L}(0,0.5,0.5)$.

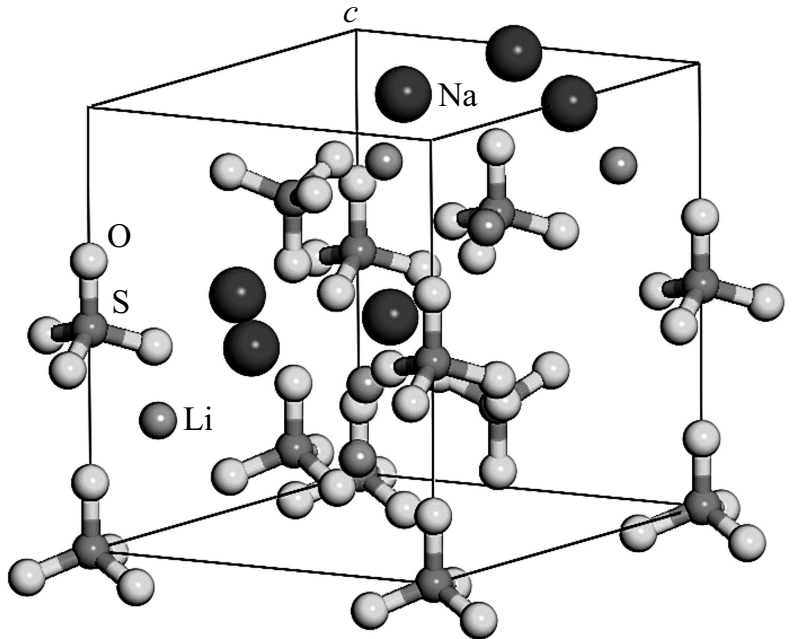

Рис. 2. Кристаллическая структура $\mathrm{LiNaSO}_{4}$ при комнатной температуре.

мальное отклонение координат $0.183 \AA$ ), тогда как GGA дает значения очень близкие к экспериментальным.

Рассчитанная зонно-энергетическая диаграмма для кристалла ЛНС представлена на рис. 3. Как видно из рисунка, энергетические уровни вершины валентной зоны (B3) характеризуются низкой дисперсией, типичной для кристаллов группы $\mathrm{ABSO}_{4}[13,18-20]$, что указывает на относительно слабую химическую связь между формирующими их группами атомов. Максимальная дисперсия $E(\mathbf{k})$ наблюдается для низких уровней зоны проводимости (ЗП) участков ЗБ $-\mathrm{A}, \mathrm{K}-\Gamma, \Gamma-\mathrm{M}$.

Вершина В3, обозначенная как $0 \mathrm{eV}$, совпадает с уровнем Ферми $E_{F}$. Запрещенная зона кристалла ЛНС является зоной прямого типа, что предусматривает прямые оптические переходы в точке Г ЗБ (рис. 3). Однако вследствие низкой дисперсности энергетических уровней возможны также непрямые переходы с участием фононов, которые не учитываются в расчетах. Полученные значения ширины запрещенной зоны $\left(E_{g}=5.49 \mathrm{eV}(\mathrm{GGA})\right.$ и $\left.E_{g}=5.89 \mathrm{eV}(\mathrm{LDA})\right)$ удовлетворительно совпадают с найденными ранее экспериментально на основе исследований края фундаментального поглощения $\left(E_{g}=4.7 \mathrm{eV}\right)$ [9] и пика фотолюминесценции $\left(E_{g}=5 \mathrm{eV}\right)[21]$. Стоит отметить, что завышение ширины запрещенной зоны является нетипичным для расчетов на базе DFT. Можно предположить, что низкое $E_{g}$ в реальном кристалле вызвано дополнительными уровнями вблизи дна зоны проводимости.

При сравнении полученных результатов с данными расчетов для кристаллов $\mathrm{LiKSO}_{4}\left(E_{g}=5.8 \mathrm{eV}\right)$ [18], $\mathrm{LiRbSO}_{4} \quad\left(E_{g}=5.16 \mathrm{eV}\right) \quad[20] \quad$ и $\quad \mathrm{LiNH}_{4} \mathrm{SO}_{4} \quad\left(E_{g}=\right.$ $=5.32 \mathrm{eV}$ ) [12] можно увидеть, что ширина запрещенной зоны кристалла $\mathrm{LiNaSO}_{4}$ меньше, чем в $\mathrm{LiKSO}_{4}$, и больше, чем в $\mathrm{LiNH}_{4} \mathrm{SO}_{4}$. В общем $E_{g}$ возрастает при катионном замещении $\mathrm{Rb} \rightarrow \mathrm{NH}_{4} \rightarrow \mathrm{Na} \rightarrow \mathrm{K}$ на 0.16 , 0.17 и $0.31 \mathrm{eV}$ соответственно. 
Параметры решетки и объем элементарной ячейки кристалла $\mathrm{LiNaSO}_{4}$, полученные экспериментально и рассчитанные из первых принципов, пространственная группа $P 31 c, Z=6$

\begin{tabular}{|c|c|c|c|c|c|}
\hline \multirow{2}{*}{$\begin{array}{c}\text { Параметры } \\
\text { элементарной ячейки } \\
\text { кристалла } \mathrm{LiNaSO}_{4}\end{array}$} & \multirow[t]{2}{*}{ Эксперимент } & \multicolumn{2}{|c|}{ Расчет } & \multicolumn{2}{|c|}{$\begin{array}{c}\text { Отклонение от } \\
\text { экспериментальных данных }\end{array}$} \\
\hline & & GGA & LDA & GGA & LDA \\
\hline $\begin{array}{l}a=b, \AA \\
c, \AA \\
c / a \\
\text { Объем } V, \AA^{3}\end{array}$ & $\begin{array}{l}7.6299(2) \\
9.8597(2) \\
1.2922 \\
497.09(3)\end{array}$ & $\begin{array}{c}7.6190 \\
9.8490 \\
1.3116 \\
77495.1294\end{array}$ & $\begin{array}{c}7.4459 \\
9.7027 \\
1.3031 \\
465.8624\end{array}$ & $\begin{array}{l}-0.1 \% \\
-0.1 \% \\
-1.5 \% \\
-0.4 \%\end{array}$ & $\begin{array}{l}-2.4 \% \\
-1.6 \% \\
-0.9 \% \\
-6.3 \%\end{array}$ \\
\hline
\end{tabular}
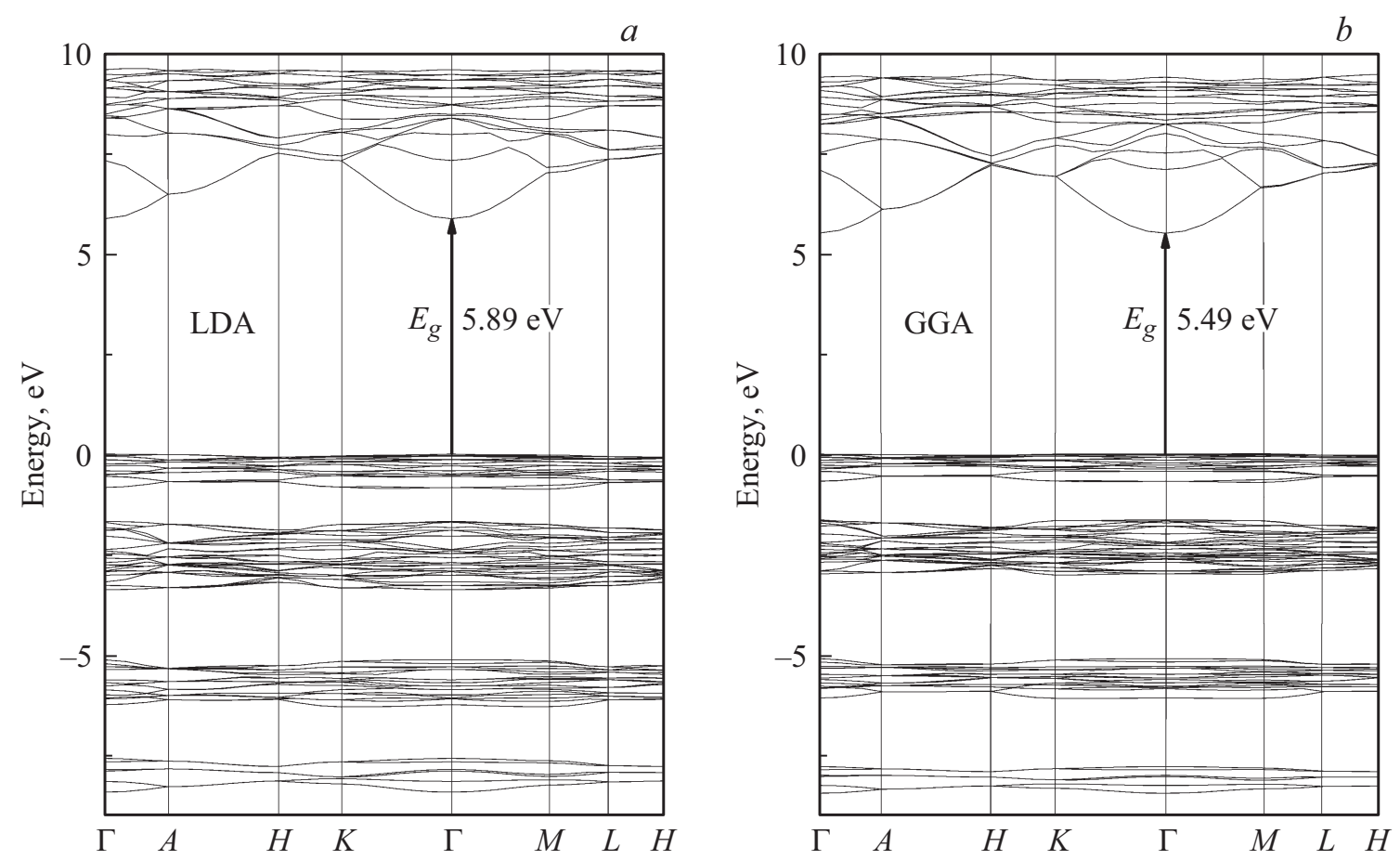

Рис. 3. Рассчитанная зонно-энергетическая диаграмма для кристалла $\mathrm{LiNaSO}_{4}$ с использованием функционалов LDA (a) и GGA $(b)$.

Спектральные зависимости полной и парциальных электронных плотностей состояний для атомов с соответствующими орбитальными моментами для кристалла $\mathrm{LiNaSO}_{4}$ представлены на рис. 4. Характерной особенностью данного распределения является то, что верхние валентные зоны (от -3.8 до $0 \mathrm{eV}$ ) почти полностью образованы $p$-электронами кислорода анионных комплексов $\mathrm{SO}_{4}^{2-}$. В расположенных глубже уровнях валентной зоны в диапазоне от -8.5 до $-5 \mathrm{eV}$, кроме $p$-состояний кислорода, значительный вклад дают $s$ состояния кислорода и $p$-состояния серы.

Валентные зоны от -17.5 до $-20.5 \mathrm{eV}$ содержат две подзоны: одна образована преимущественно анионными комплексами $\mathrm{SO}_{4}$ (s-состояниями кислорода гибридизованными с $p$-состояниями серы), а другая - $p$-состояниями натрия. Уровень энергии вблизи $-22.5 \mathrm{eV}$ формируется, главным образом, за счет $s$-состояний серы $\mathrm{S}$ и кислорода О, $s$-состояния ли- тия и натрия проявляются в В3 при энергиях -41.4 и $-47.5 \mathrm{eV}$ соответственно.

Дно зоны проводимости (ЗП) кристалла сформировано в основном $s$-состояниями лития и натрия. При энергиях $E>6 \mathrm{eV}$ ярко выраженными являются $p$-состояния серы, кислорода и натрия.

\section{Оптические спектры кристаллов $\mathrm{LiNaSO}_{4}$}

Для исследования оптических свойств удобно использовать комплексную диэлектрическую функцию $\varepsilon(\omega)$. Ее мнимая часть $\varepsilon_{2}(\omega)$ может быть рассчитана из элементов матрицы импульсов между занятыми и незанятыми волновыми функциями [22]. Действительную часть диэлектрической функции $\varepsilon_{1}(\omega)$ можно получить из мнимой с помощью известного соотношения КрамерсаКронига [23]. 


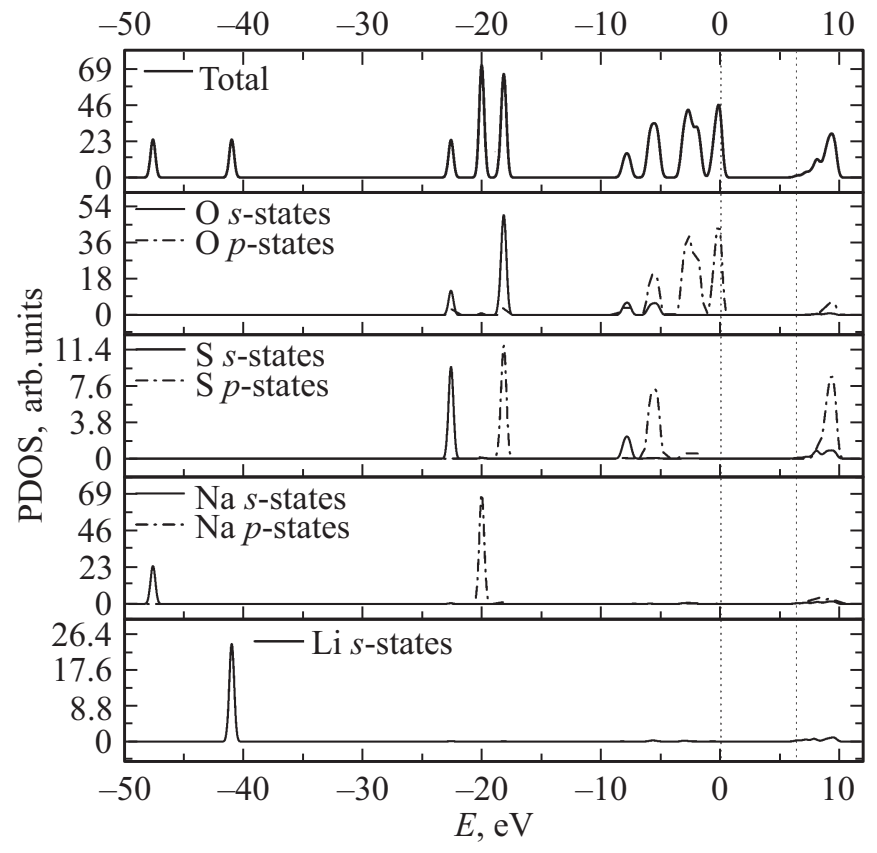

Рис. 4. Распределение электронной плотности состояний для кристаллов $\mathrm{LiNaSO}_{4}$.

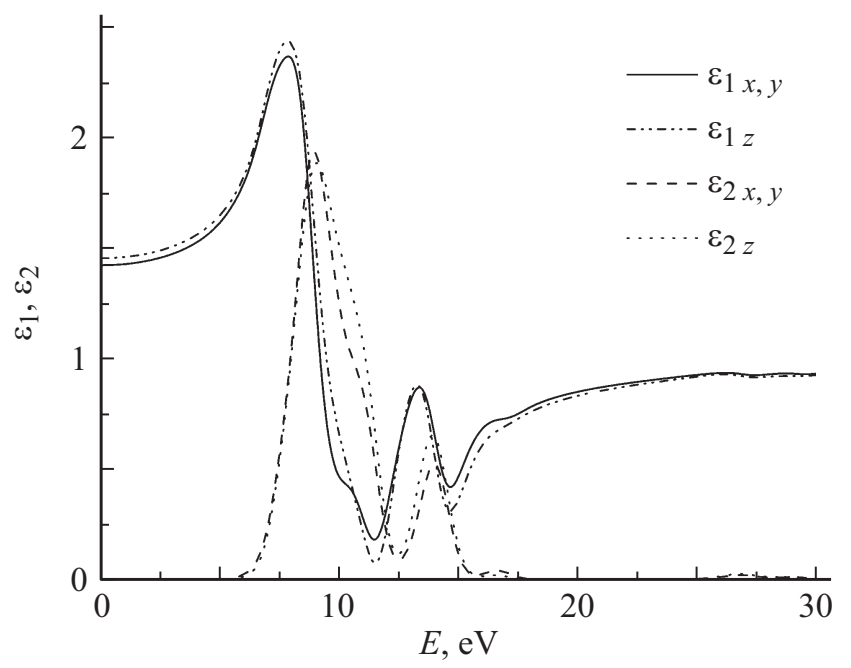

Рис. 5. Спектры действительной и мнимой частей диэлектрической функции, рассчитанные для трех главных кристаллооптических направлений $\mathrm{LiNaSO}_{4}(\mathrm{GGA})$.

Вычисленные действительная и мнимая части диэлектрической функции для монокристалла $\mathrm{LiNaSO}_{4}$ для энергий до $30 \mathrm{eV}$ представлены на рис. 5. Из кривой мнимой части диэлектрической функции можно увидеть, что первая оптическая критическая точка диэлектрической функции, которой является край фундаментального поглощения, возникает при энергии $\sim 5.5 \mathrm{eV}$. При увеличении энергии наблюдается типичный быстрый рост $\varepsilon_{2}(\omega)$. Анизотропия диэлектрической функции равна $\delta \varepsilon=0.022$.
Используя соотношение

$$
n=\sqrt{\frac{\left(\varepsilon_{1}^{2}+\varepsilon_{2}^{2}\right)^{1 / 2}+\varepsilon_{1}}{2}}, \quad k=\sqrt{\frac{\left(\varepsilon_{1}^{2}+\varepsilon_{2}^{2}\right)^{1 / 2}-\varepsilon_{1}}{2}},
$$

на основе рассчитанных спектров действительной и мнимой частей диэлектрической функции получены спектральные зависимости показателей преломления и коэффициента поглощения (рис. 6).

Теоретическая дисперсия показателей преломления для кристалла $\mathrm{LiNaSO}_{4}$ для видимой области спектра показана на рис. 7. Как видно из рисунка, показатели преломления удовлетворяют соотношению $n_{z}>n_{x, y}$ для всего представленного диапазона длин волн. Качественно полученные кривые совпадают с экспериментально измеренными (вставка на рис. 7) [10]. Теоретически полученные значения показателей преломления меньше (в пределах 20\%) экспериментальных. Такое отклонение

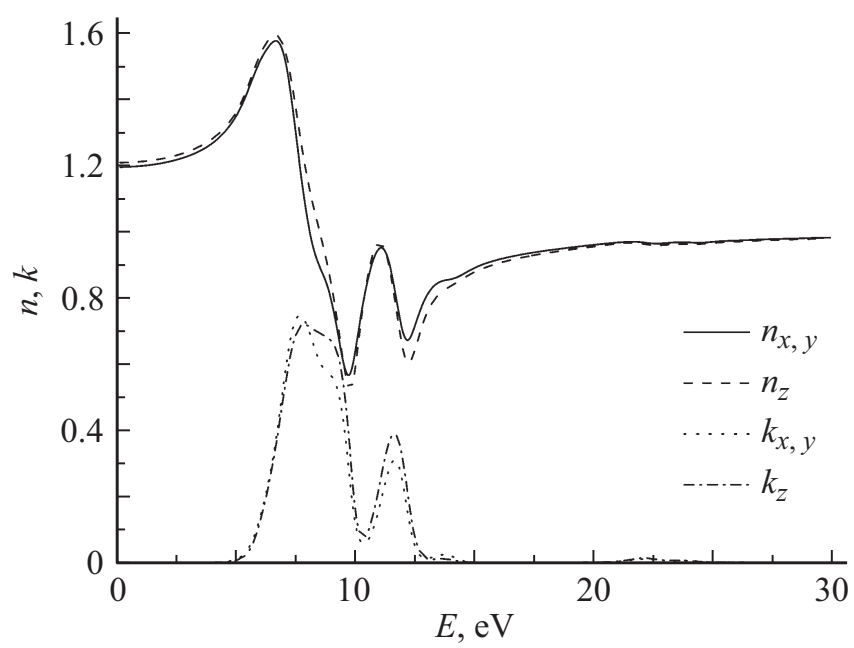

Рис. 6. Спектры показателя преломления и коэффициента поглощения, рассчитанные для трех главных кристаллооптических направлений $\mathrm{LiNaSO}_{4}$ (GGA).

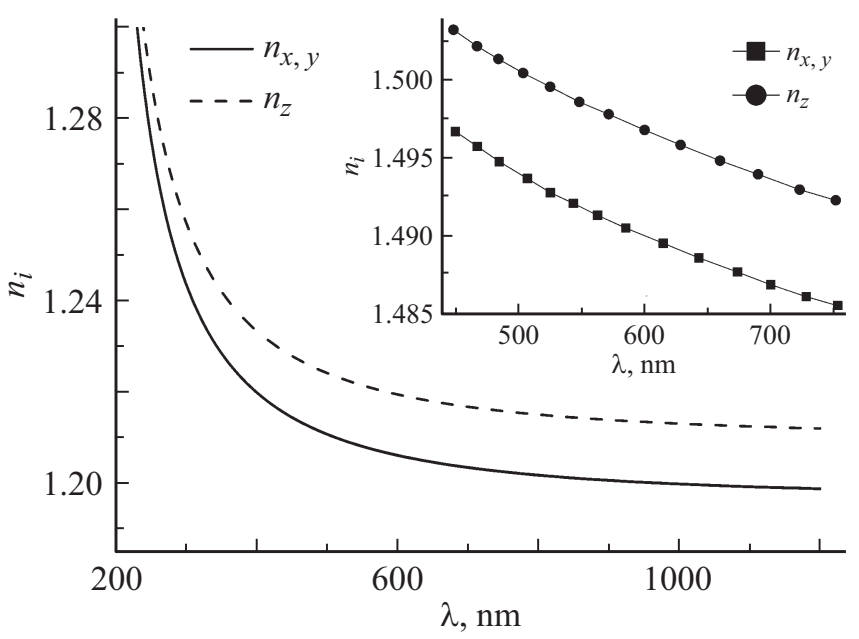

Рис. 7. Рассчитанная и экспериментальная (вставка) дисперсия показателей преломления кристалла $\mathrm{LiNaSO}_{4}$. 
может быть вызвано негативным вкладом инфракрасного поглощения в кристалле, которое не учитывается в программе CASTEP.

Однако видно, что расчетами получено очень хорошее воспроизведение дисперсионных изменений показателей преломления. Так, для зависимостей $n_{x, y}(\lambda)$ и $n_{z}(\lambda)$ величины $d n_{i} / d \lambda$ практически одинаковы и составляют $-8.8 \cdot 10^{-5} \mathrm{~nm}^{-1}(\lambda=450 \mathrm{~nm})$ и $-1.7 \cdot 10^{-5} \mathrm{~nm}^{-1}$ $(\lambda=750 \mathrm{~nm})$ для рассчитанных и $-5.5 \cdot 10^{-5} \mathrm{~nm}^{-1}$ $(\lambda=450 \mathrm{~nm})$ и $-2.5 \cdot 10^{-5} \mathrm{~nm}^{-1}(\lambda=750 \mathrm{~nm})$ для экспериментальных кривых.

\section{Выводы}

Расчеты зонно-энергетической структуры и оптических спектров кристалла $\mathrm{LiNaSO}_{4}$ в рамках теории функционала плотности позволяют сделать следующие выводы.

Оптимизированные структурные параметры кристалла ЛНС близки к экспериментальным. Максимальная дисперсия энергетических уровней $E(\mathbf{k})$ наблюдается для самых низких уровней зоны проводимости на участках $\Gamma \rightarrow \mathrm{A}, \mathrm{K} \rightarrow \Gamma$ и $\Gamma \rightarrow \mathrm{M} \mathrm{ЗБ,} \mathrm{а} \mathrm{вершина} \mathrm{валентной} \mathrm{зоны}$ и дно зоны проводимости кристалла расположены в ее центре. Ширина запрещенной зоны $\mathrm{LiNaSO}_{4}$ в рамках приближения GGA составляет $5.49 \mathrm{~nm}$ и близка по значению к соответствующим для $\mathrm{LiKSO}_{4}$ и $\mathrm{LiNH}_{4} \mathrm{SO}_{4}$. В вершине валентной зоны кристалла главную роль играют $p$-электроны кислорода, а на дне зоны проводимости - $s$-состояния лития и натрия.

Из рассчитанных спектров действительной и мнимой частей диэлектрической функции построены спектральные зависимости показателя преломления и коэффициента поглощения кристалла $\mathrm{LiNH}_{4} \mathrm{SO}_{4}$. Установлено, что теоретические значения $n_{i}$ несколько меньше средних экспериментальных значений, однако хорошо воспроизводят характер их дисперсионных зависимостей.

Расчеты проведены с использованием программного пакета Materials Studio 8.0 в суперкомпютерном центре Вроцлавского университета технологий (WCSS). Работа поддержана исследовательским проектом Академии им. Яна Длугоша „Молодые ученые 2017“، (DSM/WMP/5542/2017 - П.А. Щепанский и DSM/WMP/5536/2017 — М.Я. Рудиш).

\section{Список литературы}

[1] Freiheit H.-Ch., Kroll H., Putnis A. // Zeit. Krist. 1998. V. 213. N 11. P. 575.

[2] Morosin B., Smith D.L. // Acta Cryst. 1967. V. 22. N 6. P. 906.

[3] Karppinen M. // Acta Cryst. B. 2015. V. 71. N 3. P. 334.

[4] Chen R.H., Tseng Chaw-Ming, Shern C.S., Fukami T. // Solid State Ionics. 2010. V. 181. N 19. P. 877.

[5] Schroeder K., Kvist A., Ljungmark H. // Zeit. Naturforsch. A. 1972. V. 27a. P. 1252.
[6] Gupta K.K., Kadam R.M., Dhoble N.S., Lochab S.P., Dhoble S.J. // Phys. Chem. Chem. Phys. 2018. V. 20. N 3. P. 1540.

[7] Shakhovoy R.A., Rakhmatullin A., Deschamps M., SarouKanian V., Bessada C. // J. Phys.: Condens. Matter. 2012. V. 28. N 17. P. 176003.

[8] Teeters D., Frech R. // J. Chem. Phys. 1982. V. 76. N 2. P. 799.

[9] Abdulwahab A.M. // J. Phys. Chem. Solids. 2016. V. 99. P. 11.

[10] Shchepanskyi P.A., Kushnir O.S., Stadnyk V.Yo., Fedorchuk A.O. // Ukr. J. Phys. Opt. 2018. (in press)

[11] Стадник В.И., Кушнир О.С., Брезвин Р.С., Габа В.М. // Опт. и спектр. 2009. Т. 106. N 4. С. 685; Stadnyk V.I., Kushnir O.S., Brezvin R.S., Gaba V.M. // Opt. Spectr. 2009. V. 106. N 4. P. 614.

[12] Rudysh M.Ya., Brik M.G., Stadnyk V.Yo., Brezvin R.S., Shchepanskyi P.A, Fedorchuk A.O., Khyzhun O.Y., Kityk I.V., Piasecki M. // Physica B. 2018. V. 528. P. 37.

[13] Shchepanskyi P.A., Kushnir O.S., Stadnyk V.Yo., Fedorchuk A.O., Rudysh M.Ya., Brezvin R.S., Demchenko P.Yu., Krymus A.S. // Ukr. J. Phys. Opt. 2017. V. 18. N 4. P. 187.

[14] Henry C.H. // Phys. Rev. 1966. V. 143. N 2. P. 627.

[15] Bäumer Ch., Berben D., Buse K., Hesse H., Imbrock J. // Appl. Phys. Lett. 2003. V. 82. P. 2248.

[16] Romanyuk M.O., Andriyevsky B., Kostetsky O., Romanyuk M.M., Stadnyk V.// Cond. Matter. Phys. 2002. V. 5. N 3 (31). P. 579.

[17] Clark S.J., Segall M.D., Pickard C.J., Hasnip P.J., Probert M.J., Refson K., Payne M.C. // Zeit. Krist. 2005. V. 220. N 5-6. P. 567.

[18] Kityk I.V., Kasperczyk J., Andrievskii B.V. // Phys. Lett. A. 1996. V. 216. N 1-5. P. 161.

[19] Shchepanskyi P.A., Gaba V.M., Stadnyk V.Yo., Rudysh M.Ya., Piasecki M., Brezvin R.S. // Acta Phys. Pol. A. 2018. V. 138. (in press)

[20] Стадник В.И., Андриевский Б.В., Карплюк Л.Т., Онуфбрив O.P. // Опт. и спектр. 2016. Т. 121. N 2. С. 306; Stadnyk V.Yo,Andrievskii B.V., Karplyuk L.T., Onufriv O.R. // Opt. Spectrosc. 2016. V. 121. N 2. P. 283.

[21] Puppalwar S.P., Dhoble S.J. // J. Lumin. 2013. V. 137. P. 245.

[22] Guo X.G., Chen X.S., Lu W. // Solid State Commun. 2003. V. 126. P. 441.

[23] Dressel M., Gompf B., Faltermeier D., Tripathi A.K., Pflaum J., Schubert M. // Opt. Express. 2008. V. 16. P. 19770. 